\title{
The Bishop-Phelps-Bollobás Property: a Finite-Dimensional Approach
}

\author{
by \\ María D. Acosta, Julio Becerra Guerrero, Domingo García, \\ Sun Kwang KIM, and Manuel Maestre
}

\begin{abstract}
Our goal is to study the Bishop-Phelps-Bollobás property for operators from $c_{0}$ into a Banach space. We first characterize those Banach spaces $Y$ for which the BishopPhelps-Bollobás property holds for $\left(\ell_{\infty}^{3}, Y\right)$. Examples of spaces satisfying this condition are provided.
\end{abstract}

2010 Mathematics Subject Classification: Primary 46B20; Secondary 46B28, 47B99.

Keywords: Bishop-Phelps-Bollobás theorem, Banach space, $c_{0}$.

\section{$\S 1$. Introduction}

The Bishop-Phelps theorem [5] states that every continuous linear functional on a Banach space can be approximated by norm attaining functionals. Shortly after this assertion was proved, Bollobás gave the following "quantitative version" of that result which is called the Bishop-Phelps-Bollobás theorem [6].

Communicated by N. Ozawa. Received June 16. 2014. Revised August 28. 2014.

M. D. Acosta: Departamento de Análisis Matemático, Facultad de Ciencias, Universidad de Granada, 18071 Granada, Spain;

e-mail: dacosta@ugr.es

J. Becerra Guerrero: Departamento de Análisis Matemático, Facultad de Ciencias, Universidad de Granada, 18071 Granada, Spain;

e-mail: juliobg@ugr.es

D. García: Departamento de Análisis Matemático, Universidad de Valencia,

Doctor Moliner 50, 46100 Burjasot (Valencia), Spain;

e-mail: domingo.garcia@uv.es

S. K. Kim: Department of Mathematics, Kyonggi University, Suwon 443-760,

Republic of Korea;

e-mail: sunkwang@kgu.ac.kr

M. Maestre: Departamento de Análisis Matemático, Universidad de Valencia, Doctor Moliner 50, 46100 Burjasot (Valencia), Spain;

e-mail: manuel.maestre@uv.es 
Theorem 1.1 (Bishop-Phelps-Bollobás Theorem, [7, Theorem 16.1]). Let $X$ be a Banach space and $0<\varepsilon<1$. Given $x \in B_{X}$ and $x^{*} \in S_{X^{*}}$ with $\left|1-x^{*}(x)\right|<$ $\varepsilon^{2} / 4$, there are elements $y \in S_{X}$ and $y^{*} \in S_{X^{*}}$ such that $y^{*}(y)=1,\|y-x\|<\varepsilon$ and $\left\|y^{*}-x^{*}\right\|<\varepsilon$.

After the Bishop-Phelps theorem was proved, a lot of attention was devoted to extending it to operators. More recently the study of extensions of BishopPhelps-Bollobás theorem to operators was initiated by Acosta, Aron, García and Maestre [2].

Definition $1.2([2])$. Let $X$ and $Y$ be either real or complex Banach spaces. The pair $(X, Y)$ is said to have the Bishop-Phelps-Bollobás property for operators (BPBp) if for every $\varepsilon>0$ there exists $\eta(\varepsilon)>0$ such that for every $T \in S_{\mathcal{L}(X, Y)}$, if $x_{0} \in S_{X}$ is such that

$$
\left\|T x_{0}\right\|>1-\eta(\varepsilon)
$$

then there exist an element $u_{0}$ in $S_{X}$ and an operator $S$ in $S_{\mathcal{L}(X, Y)}$ satisfying the following conditions:

$$
\left\|S u_{0}\right\|=1, \quad\left\|u_{0}-x_{0}\right\|<\varepsilon \quad \text { and } \quad\|S-T\|<\varepsilon .
$$

Acosta, Aron, García and Maestre [2] showed that the pair $(X, Y)$ has the BPBp whenever $X$ and $Y$ are finite-dimensional spaces. They also proved that if $Y$ has a certain geometric property (property $\beta$ of Lindenstrauss), then $(X, Y)$ has the BPBp for every Banach space $X$. They also characterized the Banach spaces $Y$ such that $\left(\ell_{1}, Y\right)$ has the BPBp.

However, the case of $X=c_{0}$ is quite different and seems to be much more difficult. Acosta et al. [2] showed that $\left(\ell_{\infty}^{n}, Y\right)$ has the BPBp for every positive integer $n$ whenever $Y$ is uniformly convex. Recently $\operatorname{Kim}[8]$ proved that $\left(c_{0}, Y\right)$ also has this property under the same assumption. Aron at al. [3] studied this question for $Y=\mathcal{C}_{0}(L)$, where $L$ is a locally compact Hausdorff topological space. They showed that the couple $\left(X, \mathcal{C}_{0}(L)\right)$ has the BPBp if $X$ is Asplund. This result was extended by Cascales, Guirao and Kadets [4] to uniform algebras. Since $c_{0}$ is Asplund it follows that $\left(c_{0}, \mathcal{C}_{0}(L)\right)$ has the BPBp. For more results where the domain space is some space $\mathcal{C}(K)$ see also [1] and [9]. However until now there is no characterization of the spaces $Y$ such that $\left(c_{0}, Y\right)$ has the BPBp. In this paper, we approach this problem by using appropriate finite-dimensional spaces. From now on, we only consider real normed spaces.

In Section 2, we characterize the Banach spaces $Y$ such that $\left(\ell_{\infty}^{3}, Y\right)$ has the BPBp. We also prove that uniformly convex spaces, $\mathcal{C}(K)$ and $L_{1}(\mu)$ always have this property. We show that $\left(\ell_{\infty}^{3}, \mathcal{C}_{0}(L, X)\right)$ has this property if and only if $\left(\ell_{\infty}^{3}, X\right)$ does, whenever $L$ is any nonempty locally compact Hausdorff space. 


\section{§2. The Bishop-Phelps-Bollobás property for operators from $\ell_{\infty}^{3}$ into any Banach space}

As usual, we denote by $\ell_{\infty}^{n}$ the space $\mathbb{R}^{n}$ endowed with the supremum norm. Since $\ell_{\infty}^{2}$ is isometrically isomorphic to $\ell_{1}^{2}$, it is an easy consequence of Acosta et al. [2, Theorem 4.1] that $\left(\ell_{\infty}^{2}, X\right)$ has the BPBp if and only if $X$ has the approximate hyperplane series property for convex combinations of two elements. However, $\ell_{\infty}^{3}$ is not isometric to $\ell_{1}^{3}$, and so we cannot get a parallel result.

In order to characterize the Banach spaces $X$ such that $\left(\ell_{\infty}^{3}, X\right)$ has the BPBp we introduce the following property. This condition resembles the restricted 3-ball property which is used to characterize when a subspace of a Banach space is an M-ideal.

Definition 2.1. A Banach space $X$ has the approximate hyperplane sum property for $\ell_{\infty}^{3}\left(\right.$ AHSP- $\left.\ell_{\infty}^{3}\right)$ if for every $\varepsilon>0$ there is $\delta(\varepsilon)>0$ satisfying the following.

For every subset $\left\{x_{i}: i \leq 3\right\} \subset B_{X}$ with $\left\|x_{1}+x_{2}-x_{3}\right\| \leq 1$, if there exist a nonempty subset $C$ of $\{1,2,3\}$ and $x^{*} \in S_{X^{*}}$ such that $x^{*}\left(x_{i}\right)>1-\delta(\varepsilon)$ for every $i \in C$, then there exists $\left\{z_{i}: i \leq 3\right\} \subset B_{X}$ with $\left\|z_{1}+z_{2}-z_{3}\right\| \leq 1$ satisfying $\left\|z_{i}-x_{i}\right\|<\varepsilon$ for every $i \leq 3$ and $\left\|\sum_{i \in C} z_{i}\right\|=|C|$.

We try to explain the idea behind the property defined above. It is trivially satisfied that the unit ball of $\ell_{\infty}^{3}$ is the absolutely convex hull of four vectors. Indeed one of these vectors can be written as a linear combination of the rest with scalars $\{1,1,-1\}$. In this way, the set $\left\{x_{i}: i \leq 3\right\}$ in Definition 2.1 can be identified with an operator $T$ from $\ell_{\infty}^{3}$ into $X$ whose norm is close to 1 . Moreover the assumption implies that $T$ is close to its norm at some convex combination $c_{1}$ of the elements $\left\{x_{i}: i \leq 3\right\}$ that belong to the unit sphere. The elements $\left\{z_{i}: i \leq 3\right\}$ will be associated to a new operator $S$ close to $T$ and attaining its norm at some convex combination $c_{2}$ of $\left\{z_{i}: i \leq 3\right\}$, which is close to $c_{1}$. This is why we assume that several elements of the set $\left\{z_{i}: i \leq 3\right\}$ have the same supporting hyperplane. We also notice that condition (3) in Proposition 2.2 below resembles the definition of the approximate hyperplane series property (see [2, Definition 1.1]).

It is very easy to check directly that $\mathbb{R}$ has the above property. In any case, later we will show more general results and provide several examples.

First, recall that a subset $B \subset B_{X^{*}}$ is 1-norming if $\|x\|=\sup _{x^{*} \in B}\left|x^{*}(x)\right|$ for every $x \in X$.

The following characterization will be useful.

Proposition 2.2. Let $X$ be a Banach space. The following conditions are equivalent: 
(1) $X$ has the approximate hyperplane sum property for $\ell_{\infty}^{3}$.

(2) There is a 1-norming subset $B \subset S_{X^{*}}$ such that the condition stated in Definition 2.1 is satisfied for every $x^{*} \in B$.

(3) For every $\varepsilon>0$ there exists $\eta(\varepsilon)>0$ such that for every subset $\left\{x_{i}: i \leq 3\right\}$ $\subset B_{X}$ with $\left\|x_{1}+x_{2}-x_{3}\right\| \leq 1$ and every convex combination $\sum_{i=1}^{3} \alpha_{i} x_{i}$ satisfying

$$
\left\|\sum_{i=1}^{3} \alpha_{i} x_{i}\right\|>1-\eta(\varepsilon),
$$

there exist $A \subset\{1,2,3\},\left\{z_{i}: i \leq 3\right\} \subset B_{X}$ and $x^{*} \in S_{X^{*}}$ such that

(i) $\sum_{i \in A} \alpha_{i}>1-\varepsilon$,

(ii) $\left\|z_{i}-x_{i}\right\|<\varepsilon$ for all $i \leq 3$,

(iii) $x^{*}\left(z_{i}\right)=1$ for all $i \in A$,

(iv) $\left\|z_{1}+z_{2}-z_{3}\right\| \leq 1$.

Proof. Clearly (1) implies (2). Assume that $X$ satisfies (2). Given $0<\varepsilon<1$, let $0<\delta(\varepsilon)<1$ satisfy the condition of Definition 2.1 for all $x^{*} \in B$. Consider a subset $\left\{x_{i}: i \leq 3\right\} \subset B_{X}$ with $\left\|x_{1}+x_{2}-x_{3}\right\| \leq 1$ and three nonnegative real numbers $\alpha_{i}$ for $1 \leq i \leq 3$ with $\sum_{i=1}^{3} \alpha_{i}=1$ also satisfying

$$
\left\|\sum_{i=1}^{3} \alpha_{i} x_{i}\right\|>1-\varepsilon \delta(\varepsilon) .
$$

Then there exists $x^{*} \in B$ such that

$$
\sum_{i=1}^{3} \alpha_{i} x^{*}\left(x_{i}\right)>1-\varepsilon \delta(\varepsilon) .
$$

By [2, Lemma 3.3] the set $A:=\left\{i \leq 3: x^{*}\left(x_{i}\right)>1-\delta(\varepsilon)\right\}$ satisfies the estimate

$$
\sum_{i \in A} \alpha_{i}>1-\varepsilon .
$$

By assumption there exists $\left\{z_{i}: i \leq 3\right\} \subset B_{X}$ satisfying

(ii) $\left\|z_{i}-x_{i}\right\|<\varepsilon$ for all $i \leq 3$,

(iii) $\left\|\sum_{i \in A} z_{i}\right\|=|A|$,

(iv) $\left\|z_{1}+z_{2}-z_{3}\right\| \leq 1$.

Hence $X$ satisfies the conditions stated in (3) for $\eta(\varepsilon)=\varepsilon \delta(\varepsilon)$.

Now we assume that $X$ has the property in (3). Given $0<\varepsilon<1 / 3$, let $\eta(\varepsilon)>0$ satisfy conditions (i) to (iv). We are going to check that $\delta=\eta(\varepsilon)$ satisfies the condition of Definition 2.1. Let $\left\{x_{i}: i \leq 3\right\} \subset B_{X}$ satisfy $\left\|x_{1}+x_{2}-x_{3}\right\| \leq 1$, 
let $x^{*} \in S_{X^{*}}$ and let $C \subset\{1,2,3\}$ be such that $x^{*}\left(x_{i}\right)>1-\eta(\varepsilon)$ for every $i \in C$. We distinguish several cases.

Case 1: $C=\{1,2,3\}$. We take $\alpha_{1}=\alpha_{2}=\alpha_{3}=1 / 3$. Obviously $\sum_{i=1}^{3} \alpha_{i}=1$ and

$$
\left\|\sum_{i=1}^{3} \alpha_{i} x_{i}\right\| \geq \frac{1}{3} \sum_{i=1}^{3} x^{*}\left(x_{i}\right)>1-\eta(\varepsilon) .
$$

Thus there exist $A \subset\{1,2,3\},\left\{z_{i}: i \leq 3\right\} \subset S_{X}$ and $z^{*} \in S_{X^{*}}$ such that

(i) $\sum_{i \in A} \alpha_{i}>1-\varepsilon>2 / 3$,

(ii) $\left\|z_{i}-x_{i}\right\|<\varepsilon$ for all $i$,

(iii) $z^{*}\left(z_{i}\right)=1$ for all $i \in A$,

(iv) $\left\|z_{1}+z_{2}-z_{3}\right\| \leq 1$.

In view of condition (i) we have $A=\{1,2,3\}=C$ and so $X$ satisfies the AHSP- $\ell_{\infty}^{3}$.

Case 2: $C$ has two elements. For instance, if $C=\{1,2\}$, we take $\alpha_{1}=\alpha_{2}$ $=1 / 2$ and $\alpha_{3}=0$. We have

$$
\left\|\sum_{i=1}^{3} \alpha_{i} x_{i}\right\| \geq \frac{1}{2} \sum_{i=1}^{2} x^{*}\left(x_{i}\right)>1-\eta(\varepsilon) .
$$

Therefore, there exist $A \subset\{1,2,3\},\left(z_{i}\right)_{i=1}^{3} \subset S_{X}$ and $y^{*} \in S_{X^{*}}$ such that

(i) $\sum_{i \in A} \alpha_{i}>1-\varepsilon>2 / 3$,

(ii) $\left\|z_{i}-x_{i}\right\|<\varepsilon$ for all $i$,

(iii) $y^{*}\left(z_{i}\right)=1$ for all $i \in A$,

(iv) $\left\|z_{1}+z_{2}-z_{3}\right\| \leq 1$.

Again, by (i) we have $A \supset\{1,2\}=C$ and the condition in Definition 2.1 is satisfied.

If $C=\{1,3\}$ or $C=\{2,3\}$ we can argue in a similar way.

Case 3: $C=\{k\}$. We take $\alpha_{k}=1$ and $\alpha_{i}=0$ for $i \in\{1,2,3\} \backslash\{k\}$. It is straightforward that analogous arguments to those above show that $X$ has the approximate hyperplane sum property for $\ell_{\infty}^{3}$.

Let us remark that we have checked that the function $\delta(\varepsilon)=\eta(\varepsilon)$ satisfies the condition of Definition 2.1, where $\eta$ is the function appearing in condition (3).

Recall that a Banach space $X$ is uniformly convex if for every $\varepsilon>0$ there is $0<\delta<1$ such that

$$
u, v \in B_{X}, \frac{\|u+v\|}{2}>1-\delta \Rightarrow\|u-v\|<\varepsilon .
$$

In that case, the modulus of convexity of $X$ is the function defined by

$$
\delta(\varepsilon):=\inf \left\{1-\|u+v\| / 2: u, v \in B_{X},\|u-v\| \geq \varepsilon\right\} .
$$


Proposition 2.3. Every uniformly convex space has the approximate hyperplane sum property for $\ell_{\infty}^{3}$.

Proof. Let $X$ be uniformly convex with modulus of convexity $\delta$. Given $0<\varepsilon<1$, take $0<t=\min \{\delta(\varepsilon) / 2,2 \varepsilon / 3\}$. Assume that $\left\{x_{i}: i \leq 3\right\} \subset B_{X}$ with $\left\|x_{1}+x_{2}-x_{3}\right\|$ $\leq 1, \emptyset \neq C \subset\{1,2,3\}$ and $x^{*} \in S_{X^{*}}$ are such that $x^{*}\left(x_{i}\right)>1-t$ for every $i \in C$. Then for all $i, j \in C$ we have

$$
1-\frac{\delta(\varepsilon)}{2} \leq 1-t<x^{*}\left(\frac{x_{i}+x_{j}}{2}\right) \leq\left\|\frac{x_{i}+x_{j}}{2}\right\| .
$$

As a consequence $\left\|x_{i}-x_{j}\right\|<\varepsilon$ for all $i, j \in C$.

If $C=\{1,2,3\}$, choose $i_{0} \in C$ and take $z_{i}=x_{i_{0}} /\left\|x_{i_{0}}\right\|$ for every $i \in C$. By the definition of the modulus of convexity it follows that $\left\|z_{i}-x_{i}\right\|<\varepsilon$ for every $i \in C$.

Assume that $C=\{1,2\}$; then

$$
2-2 t-x^{*}\left(x_{3}\right)<x^{*}\left(x_{1}+x_{2}-x_{3}\right) \leq\left\|x_{1}+x_{2}-x_{3}\right\| \leq 1 .
$$

Thus

$$
1-\delta(\varepsilon) \leq 1-2 t<x^{*}\left(x_{3}\right) .
$$

By using again the fact that $X$ is uniformly convex, if we take $z_{3}=x_{i_{0}} /\left\|x_{i_{0}}\right\|$, all the requirements in Definition 2.1 are satisfied.

If $C=\{1,3\}$, then it suffices to take $z_{2}=x_{2}$ and $z_{1}=z_{3}=x_{1} /\left\|x_{1}\right\|$. The case $C=\{2,3\}$ is analogous. Finally, consider the case where $C$ contains only one element, say $C=\{1\}$. In that case we take $z_{1}=x_{1} /\left\|x_{1}\right\|$. Hence

$$
\left\|z_{1}+x_{2}-x_{3}\right\| \leq 1+\left\|x_{1}\right\|\left(\frac{1}{\left\|x_{1}\right\|}-1\right)<1+t .
$$

If $\left\|z_{1}+x_{2}-x_{3}\right\| \leq 1$ it suffices to take $z_{i}=x_{i}$ for $i=2,3$. Otherwise, we write $s=\left\|z_{1}+x_{2}-x_{3}\right\|-1$ and we know that $0<s<t \leq 2 \varepsilon / 3$. We take $a=\frac{s}{1+s}, b=\frac{s}{2(1+s)}$ and $z_{2}=(1-a) x_{2}-b z_{1}, z_{3}=(1-a) x_{3}+b z_{1}$. We have

$$
\left\|z_{i}\right\| \leq 1-a+b=1-\frac{s}{1+s}+\frac{s}{2(1+s)} \leq 1, \quad i=2,3 .
$$

Moreover

$$
\begin{aligned}
\left\|z_{1}+z_{2}-z_{3}\right\| & =\left\|(1-2 b) z_{1}+(1-a)\left(x_{2}-x_{3}\right)\right\|=(1-a)\left\|z_{1}+x_{2}-x_{3}\right\| \\
& =\frac{1}{1+s}(1+s)=1 .
\end{aligned}
$$

Finally, for $i=2,3$ we obtain

$$
\left\|z_{i}-x_{i}\right\| \leq a+b=\frac{s}{1+s}+\frac{s}{2(1+s)}=\frac{3 s}{2(1+s)}<\frac{3 t}{2} \leq \varepsilon .
$$


It is also clear that

$$
\left\|z_{1}-x_{1}\right\|=\left\|\frac{x_{1}}{\left\|x_{1}\right\|}-x_{1}\right\|=1-\left\|x_{1}\right\|<1-t<\varepsilon .
$$

Thus in each case we have proved all the conditions in Definition 2.1.

Since $\mathbb{R}$ has the AHSP- $\ell_{\infty}^{3}$, from the next proposition it follows immediately that the space $\mathcal{C}_{0}(L)$ also has this property.

Proposition 2.4. Let $L$ be a nonempty locally compact Hausdorff topological space and $X$ a Banach space. Then $\mathcal{C}_{0}(L, X)$ has the $A H S P-\ell_{\infty}^{3}$ if and only if $X$ does.

Proof. (1) Assume that $X$ has the AHSP- $\ell_{\infty}^{3}$. Given $\varepsilon>0$ assume that $\delta$ satisfies the condition of Definition 2.1 for $\varepsilon / 2$. It suffices to show that (2) in Proposition 2.2 is satisfied for the set $B=\left\{x^{*} \circ \delta_{t}: t \in L, x^{*} \in S_{X^{*}}\right\} \subset S_{\left(\mathcal{C}_{0}(L, X)\right)^{*}}$.

Assume that $\left\{f_{i}: i \leq 3\right\} \subset B_{\mathcal{C}_{0}(L, X)}, C \subset\{1,2,3\}, z^{*} \in S_{X^{*}}$ and $t_{1} \in L$ satisfy

$$
\left\|f_{1}+f_{2}-f_{3}\right\| \leq 1 \quad \text { and } \quad z^{*}\left(f_{i}\left(t_{1}\right)\right)>1-\delta \quad \forall i \in C .
$$

By assumption there exist $\left\{z_{i}: i \leq 3\right\} \subset B_{X}$ and $x^{*} \in S_{X^{*}}$ satisfying

$$
\left\|z_{1}+z_{2}-z_{3}\right\| \leq 1, \quad\left\|z_{i}-f_{i}\left(t_{1}\right)\right\|<\varepsilon / 2 \quad \forall i \leq 3, \quad x^{*}\left(z_{i}\right)=1 \quad \forall i \in C .
$$

Consider the open neighborhood $U$ of $t_{1}$ given by

$$
U=\bigcap_{i=1}^{3}\left\{t \in L:\left\|f_{i}(t)-f_{i}\left(t_{1}\right)\right\|<\varepsilon / 2\right\} .
$$

By the Urysohn Lemma, there exists a function $\phi$ in $\mathcal{C}_{0}(L)$ whose support is contained in $U$, satisfying also $0 \leq \phi \leq 1$ and $\phi\left(t_{1}\right)=1$. Now we define three elements in $\mathcal{C}_{0}(L, X)$ by

$$
g_{i}=\phi z_{i}+(\mathbf{1}-\phi) f_{i} \quad(i \leq 3)
$$

It is clear that $g_{i}\left(t_{1}\right)=z_{i}$ for every $i \leq 3$, and for every $t \in L$ we have

$$
\left\|g_{i}(t)\right\| \leq|\phi(t)|+|1-\phi(t)|=1 .
$$

Moreover $g_{i}(t)=f_{i}(t)$ for any $t \in L \backslash U$ and $i \leq 3$. If $t \in U$, for every $i \leq 3$ we have

$$
\begin{aligned}
\left\|g_{i}(t)-f_{i}(t)\right\| & =\left\|\left(z_{i}-f_{i}(t)\right) \phi(t)\right\| \leq\left\|z_{i}-f_{i}\left(t_{1}\right)\right\|+\left\|f_{i}\left(t_{1}\right)-f_{i}(t)\right\| \\
& <\varepsilon / 2+\varepsilon / 2=\varepsilon .
\end{aligned}
$$

Hence $\left\|g_{i}-f_{i}\right\|<\varepsilon$ for every $i \leq 3$. 
Moreover,

$$
\left\|g_{1}+g_{2}-g_{3}\right\|=\left\|\phi\left(z_{1}+z_{2}-z_{3}\right)+(\mathbf{1}-\phi)\left(f_{1}+f_{2}-f_{3}\right)\right\| \leq 1 .
$$

Since $\left(x^{*} \circ \delta_{t_{1}}\right)\left(g_{i}\right)=1$ for every $i \in C$, we have proved that $\mathcal{C}_{0}(L, X)$ has the $\mathrm{AHSP}-\ell_{\infty}^{3}$.

(2) Assume now that $\mathcal{C}_{0}(L, X)$ has the AHSP- $\ell_{\infty}^{3}$. Given $\varepsilon>0$, let $\delta$ satisfy the condition of Definition 2.1 .

Suppose that $\left\{x_{i}: i \leq 3\right\} \subset B_{X}$ and for some $z^{*} \in S_{X^{*}}$ and $\emptyset \neq C \subset\{1,2,3\}$ we have

$$
\left\|x_{1}+x_{2}-x_{3}\right\| \leq 1 \quad \text { and } \quad z^{*}\left(x_{i}\right)>1-\delta \quad \forall i \in C
$$

Since $L$ is nonempty, we can choose $t_{0} \in L$. Again, by the Urysohn Lemma, we can find a continuous function $\phi: L \rightarrow[0,1]$ with compact support and $\phi\left(t_{0}\right)=1$. Observe that if $L$ is compact we can take the constant function $\mathbf{1}$ as $\phi$.

Now we define $f_{i} \in B_{\mathcal{C}_{0}(L, X)}$ by $f_{i}(t)=\phi(t) x_{i}$ for any $i \leq 3$ and $t \in L$. It is clear that $\left\|f_{1}+f_{2}-f_{3}\right\| \leq 1$ and

$$
\left(z^{*} \circ \delta_{t_{0}}\right)\left(f_{i}\right)>1-\delta \quad \forall i \in C .
$$

By assumption there exists $\left\{g_{i}: i \leq 3\right\} \subset B_{\mathcal{C}_{0}(L, X)}$ such that

$$
\left\|g_{i}-f_{i}\right\|<\varepsilon \text { for every } i \leq 3, \quad\left\|g_{1}+g_{2}-g_{3}\right\| \leq 1, \quad \text { and } \quad\left\|\sum_{i \in C} g_{i}\right\|=|C| .
$$

Hence there is $t_{1} \in L$ such that $|C|=\left\|\sum_{i \in C} g_{i}\right\|=\left\|\sum_{i \in C} g_{i}\left(t_{1}\right)\right\|$. Clearly $\left\|g_{i}\left(t_{1}\right)\right\|=1$ for every $i$ in $C$. On the other hand

$$
\left\|f_{i}\left(t_{1}\right)-g_{i}\left(t_{1}\right)\right\| \leq\left\|f_{i}-g_{i}\right\|<\varepsilon \quad \text { for every } i \leq 3 .
$$

For any $i \in C$, we have

$$
1-\varepsilon<\left\|f_{i}\left(t_{1}\right)\right\|=\phi\left(t_{1}\right)\left\|x_{i}\right\| \leq \phi\left(t_{1}\right) .
$$

Finally, we define $z_{i}=g_{i}\left(t_{1}\right)$ for $i \leq 3$, and so $\left\{z_{i}: i \leq 3\right\} \subset B_{X}$. For every $i \leq 3$ it is clear that

$$
\begin{aligned}
\left\|z_{i}-x_{i}\right\| & \leq\left\|\left(g_{i}-f_{i}\right)\left(t_{1}\right)\right\|+\left\|f_{i}\left(t_{1}\right)-x_{i}\right\| \\
& <\varepsilon+\left\|\phi\left(t_{1}\right) x_{i}-x_{i}\right\| \leq \varepsilon+1-\phi\left(t_{1}\right)<2 \varepsilon .
\end{aligned}
$$

Also

$$
\left\|z_{1}+z_{2}-z_{3}\right\| \leq\left\|g_{1}+g_{2}-g_{3}\right\| \leq 1
$$

and

$$
\left\|\sum_{i \in C} z_{i}\right\|=\left\|\sum_{i \in C} g_{i}\left(t_{1}\right)\right\|=|C| .
$$

We have proved that $X$ has the AHSP- $\ell_{\infty}^{3}$. 
Remark 2.5. We do not know whether AHSP- $\ell_{\infty}^{3}$ is stable under injective tensor products. If it were, this would extend Proposition 2.4.

Our goal now is to show that every space $L_{1}(\mu)$ has the AHSP- $\ell_{\infty}^{3}$.

Proposition 2.6. The space $\ell_{1}^{n}$ has the approximate hyperplane sum property for $\ell_{\infty}^{3}$ for every positive integer $n$. Actually, for every positive real $\varepsilon<1$, the number $\delta(\varepsilon)=\varepsilon / 48$ satisfies condition (2) in Proposition 2.2 for the set $\operatorname{Ext}\left(B_{\left(\ell_{1}^{n}\right)^{*}}\right)$.

Proof. We check the last statement. Let $x_{1}, x_{2}, x_{3}$ be elements in the unit ball of $\ell_{1}^{n}$ with $\left\|x_{1}+x_{2}-x_{3}\right\| \leq 1$ and such that there is $x^{*} \in \operatorname{Ext}\left(B_{\ell_{\infty}^{n}}\right)$ fulfilling for some subset $C$ of $\{1,2,3\}$ the condition $x^{*}\left(x_{i}\right)>1-\delta$ for every $i \in C$. By applying an isometry in $\ell_{1}^{n}$, we can always assume that $x^{*}\left(e_{k}\right)=1$ for every $k \leq n$. Hence

$$
\sum_{k=1}^{n} x_{i}(k)>1-\delta \quad \text { for every } i \in C, \quad \text { and } \quad\left\|x_{1}+x_{2}-x_{3}\right\| \leq 1 .
$$

We are going to discuss the possible cases.

Case 1: $C=\{1,2,3\}$. First, additionally assume that every $x_{i}$ is a nonnegative element in $B_{\ell_{1}^{n}}$ for $i \leq 3$. We write

$$
D=\left\{k \leq n: x_{3}(k) \leq x_{1}(k)+x_{2}(k)\right\} \quad \text { and } \quad E=\{1, \ldots, n\} \backslash D .
$$

Since

$$
1-2 \delta<\sum_{k=1}^{n}\left(x_{1}+x_{2}-x_{3}\right)(k) \leq \sum_{k \in D}\left(x_{1}+x_{2}-x_{3}\right)(k) \leq\left\|x_{1}+x_{2}-x_{3}\right\| \leq 1,
$$

we have $\left\|\left(x_{1}+x_{2}-x_{3}\right) \chi_{E}\right\|<2 \delta$.

Define $u_{3}=x_{3} \chi_{D}+\left(x_{1}+x_{2}\right) \chi_{E}$. Clearly $0 \leq u_{3} \leq x_{3}$ and so $\left\|u_{3}\right\| \leq 1$. Moreover

$$
\left\|u_{3}-x_{3}\right\|=\left\|\left(x_{1}+x_{2}-x_{3}\right) \chi_{E}\right\|<2 \delta
$$

and

$$
\left\|x_{1}+x_{2}-u_{3}\right\|=\left\|\left(x_{1}+x_{2}-x_{3}\right) \chi_{D}\right\| \leq 1 .
$$

From the definition of $u_{3}$ it follows that

$$
0 \leq u_{3} \leq x_{1}+x_{2}
$$

We write $a=\left\|x_{1}\right\|, b=\left\|x_{2}\right\|$ and $c=\left\|u_{3}\right\|$. Since $\left\|x_{1}+x_{2}-u_{3}\right\| \leq 1$ and $x_{1}+x_{2}-u_{3} \geq 0$ we have $a+b-c \leq 1$, that is,

$$
1-c \leq(1-a)+(1-b)
$$

We define

$$
z_{1}=x_{1}+(1-a) e_{1}, \quad z_{2}=x_{2}+(1-b) e_{1}, \quad z_{3}=u_{3}+(1-c) e_{1} .
$$


It is clear that $z_{i} \geq 0$ and $z_{i} \in S_{\ell_{1}^{n}}$ for every $i \leq 3$. Since $a, b>1-\delta$ and $c>1-2 \delta$, we obtain

$$
\left\|z_{i}-x_{i}\right\|<\delta \quad \text { for } i=1,2 \text { and } \quad\left\|z_{3}-u_{3}\right\|<2 \delta \text {. }
$$

Since $u_{3} \leq x_{1}+x_{2}$, in view of $(2.2)$ we obtain $z_{3} \leq z_{1}+z_{2}$. Hence

$$
\left\|z_{1}+z_{2}-z_{3}\right\|=\sum_{k=1}^{n}\left(z_{1}+z_{2}-z_{3}\right)(k)=1 .
$$

We also know that $\left\|z_{i}-x_{i}\right\|<\delta$ for $i=1,2$ and by using (2.3) and (2.1) we get

$$
\left\|z_{3}-x_{3}\right\| \leq\left\|z_{3}-u_{3}\right\|+\left\|u_{3}-x_{3}\right\|<2 \delta+2 \delta=4 \delta .
$$

Thus we have proved the desired fact for nonnegative elements in $\ell_{1}^{n}$.

Now we will prove that the condition in Definition 2.1 is satisfied in the case $C=\{1,2,3\}$ for any elements in $\ell_{1}^{n}$. Assume that $x_{i}$ for $i \leq 3$ are elements in $B_{\ell_{1}^{n}}$ satisfying

$$
\sum_{k=1}^{n} x_{i}(k)>1-\delta \quad \text { and } \quad\left\|x_{1}+x_{2}-x_{3}\right\| \leq 1 .
$$

For $i \leq 3$ we denote

$$
P_{i}=\left\{k \leq n: x_{i}(k) \geq 0\right\} \quad \text { and } \quad N_{i}=\{1, \ldots, n\} \backslash P_{i} .
$$

By assumption, for each $i \leq 3$ we have

$$
1-\delta<\sum_{k=1}^{n} x_{i}(k) \leq \sum_{k \in P_{i}} x_{i}(k) \leq\left\|x_{i}\right\| \leq 1 .
$$

So $u_{i}=x_{i} \chi_{P_{i}}$ is nonnegative and satisfies $1-\delta<\left\|u_{i}\right\| \leq 1$ and

$$
\left\|u_{i}-x_{i}\right\|=\left\|x_{i} \chi_{N_{i}}\right\| \leq 1-\left\|u_{i}\right\|<\delta \quad \forall i \leq 3 .
$$

Hence

$$
\left\|u_{1}+u_{2}-u_{3}\right\| \leq\left\|x_{1}+x_{2}-x_{3}\right\|+3 \delta \leq 1+3 \delta .
$$

Since $1-2 \delta<\sum_{k=1}^{n}\left(u_{1}+u_{2}-u_{3}\right)(k) \leq\left\|u_{1}+u_{2}-u_{3}\right\| \leq 1+3 \delta$, if we denote

$$
D=\left\{k \leq n: u_{3}(k)<\left(u_{1}+u_{2}\right)(k)\right\} \text { and } E=\{1, \ldots, n\} \backslash D,
$$

then $\sum_{k \in D}\left(u_{1}+u_{2}-u_{3}\right)(k)>1-2 \delta$.

If $\left\|u_{1}+u_{2}-u_{3}\right\| \leq 1$, then we do not change the elements $u_{1}, u_{2}$ and finish the proof using the fact proved for positive elements. If $\left\|u_{1}+u_{2}-u_{3}\right\|>1$ we write $d=\left\|u_{1}+u_{2}-u_{3}\right\|-1$. We clearly have

$$
d \leq(1+3 \delta)-1=3 \delta<1-2 \delta<\sum_{k \in D}\left(u_{1}+u_{2}-u_{3}\right)(k) .
$$


As a consequence, we can find $v_{i} \in B_{\ell_{1}^{n}}$ for $i=1,2$ such that

$$
\begin{aligned}
& 0 \leq v_{i} \chi_{D} \leq u_{i} \chi_{D}, \quad v_{i} \chi_{E}=u_{i} \chi_{E}, \\
& \left\|v_{i}-u_{i}\right\| \leq d<3 \delta, \quad u_{3} \chi_{D} \leq\left(v_{1}+v_{2}\right) \chi_{D}
\end{aligned}
$$

and also

$$
\sum_{k \in D}\left|\left(v_{1}+v_{2}-u_{3}\right)(k)\right|=\sum_{k \in D}\left(v_{1}+v_{2}-u_{3}\right)(k)=\sum_{k \in D}\left(u_{1}+u_{2}-u_{3}\right)(k)-d .
$$

From this equality we obtain

$$
\begin{aligned}
& \left\|v_{1}+v_{2}-u_{3}\right\|=\left\|\left(v_{1}+v_{2}-u_{3}\right) \chi_{D}\right\|+\left\|\left(v_{1}+v_{2}-u_{3}\right) \chi_{E}\right\| \\
& \quad=\left\|\left(u_{1}+u_{2}-u_{3}\right) \chi_{D}\right\|-d+\left\|\left(u_{1}+u_{2}-u_{3}\right) \chi_{E}\right\|=\left\|u_{1}+u_{2}-u_{3}\right\|-d=1 .
\end{aligned}
$$

In view of (2.4) and (2.6) we can also obtain

$$
\left\|v_{1}-x_{1}\right\| \leq\left\|v_{1}-u_{1}\right\|+\left\|u_{1}-x_{1}\right\|<3 \delta+\delta=4 \delta
$$

and analogously $\left\|v_{2}-x_{2}\right\|<4 \delta$. So we have

$$
\begin{aligned}
& \sum_{k=1}^{n} u_{3}(k) \geq \sum_{k=1}^{n} x_{3}(k)>1-\delta \\
& \sum_{k=1}^{n} v_{i}(k)>\sum_{k=1}^{n} x_{i}(k)-\left\|v_{i}-x_{i}\right\|>1-5 \delta \quad \text { for } i=1,2 .
\end{aligned}
$$

Let us remark that $v_{1}, v_{2}$ and $u_{3}$ are nonnegative elements in $B_{\ell_{1}^{n}}$. By the fact proved for positive elements, there are $z_{i} \in S_{\ell_{1}^{n}}$ such that

$$
\begin{aligned}
& \left\|z_{3}-u_{3}\right\|<20 \delta, \quad\left\|z_{1}+z_{2}-z_{3}\right\| \leq 1, \quad\left\|z_{1}+z_{2}+z_{3}\right\|=3, \\
& \left\|z_{i}-v_{i}\right\|<20 \delta \quad \text { for } i=1,2 .
\end{aligned}
$$

Finally, for $i=1,2$ we obtain

$$
\left\|z_{i}-x_{i}\right\| \leq\left\|z_{i}-v_{i}\right\|+\left\|v_{i}-x_{i}\right\|<20 \delta+4 \delta=24 \delta<\varepsilon .
$$

By (2.4) we have

$$
\left\|z_{3}-x_{3}\right\| \leq\left\|z_{3}-u_{3}\right\|+\left\|u_{3}-x_{3}\right\|<20 \delta+\delta=21 \delta<\varepsilon
$$

Hence the statement is proved if $C=\{1,2,3\}$.

Case 2: $|C|=2$. We have either $C=\{1,2\}, C=\{1,3\}$ or $C=\{2,3\}$. The last two cases are equivalent to each other. Hence we have only to check the first two cases.

If $C=\{1,2\}$, we have $x^{*}\left(x_{1}\right)>1-\delta$ and $x^{*}\left(x_{2}\right)>1-\delta$ too. In this case, since $\left\|x_{1}+x_{2}-x_{3}\right\| \leq 1$, we obtain $x^{*}\left(x_{3}\right)>1-2 \delta$. By the fact proved in Case 1 , 
the inequalities (2.7) and (2.8) imply that there are $z_{i} \in B_{X}(1 \leq i \leq 3)$ satisfying $\left\|z_{i}-x_{i}\right\|<48 \delta \leq \varepsilon$ for every $i \leq 3,\left\|\sum_{i=1}^{3} z_{i}\right\|=3$ and $\left\|z_{1}+z_{2}-z_{3}\right\| \leq 1$.

If $C=\{1,3\}$, then $x^{*}\left(x_{j}\right)>1-\delta$ and so $\left\|x_{j}\right\|>1-\delta$ for $j=1,3$. If we denote

$$
P_{j}=\left\{k \leq n: x_{j}(k) \geq 0\right\} \quad(j=1,3)
$$

then $u_{j}=x_{j} \chi_{P_{j}}$ is nonnegative and satisfies $1-\delta<\left\|u_{j}\right\| \leq 1$ and $\left\|u_{j}-x_{j}\right\|<\delta$ for $j=1,3$. For $j \in C$, set $v_{j}=u_{j}+\left(1-\left\|u_{j}\right\|\right) e_{1}$. We have $\left\|v_{j}-x_{j}\right\|<2 \delta<\varepsilon$, $\left\|v_{j}\right\|=1$ for $j=1,3$ and $x^{*}\left(v_{1}+v_{3}\right)=2$. If $\left\|v_{1}+x_{2}-v_{3}\right\| \leq 1$ we are done simply by taking $z_{j}=v_{j}$ for $j=1,3$ and $z_{2}=x_{2}$.

If $a=\left\|v_{1}+x_{2}-v_{3}\right\|>1$, then

$$
\left\|\frac{v_{1}}{a}+\frac{x_{2}}{a}-\frac{v_{3}}{a}\right\|=1
$$

and

$$
a=\left\|v_{1}+x_{2}-v_{3}\right\| \leq\left\|x_{1}+x_{2}-x_{3}\right\|+\left\|x_{1}-v_{1}\right\|+\left\|x_{3}-v_{3}\right\|<1+4 \delta .
$$

Let $z_{j}=v_{j} / a+(1-1 / a) e_{1}$ for $j=1,3$, and $z_{2}=x_{2} / a$. Clearly $\left\|z_{j}\right\|=1$ for $j=1,3, x^{*}\left(z_{1}+z_{3}\right)=2$ and $\left\|z_{2}\right\| \leq 1$. Moreover

$$
\left\|z_{1}+z_{2}-z_{3}\right\|=\left\|\frac{v_{1}}{a}+\frac{x_{2}}{a}-\frac{v_{3}}{a}\right\|=1 .
$$

For $j=1,3$ we have $\left\|v_{j} / a-v_{j}\right\|=1-1 / a$ and so

$$
\left\|z_{j}-x_{j}\right\| \leq\left\|z_{j}-\frac{v_{j}}{a}\right\|+\left\|\frac{v_{j}}{a}-v_{j}\right\|+\left\|v_{j}-x_{j}\right\|<2\left(1-\frac{1}{a}\right)+2 \delta<10 \delta<\varepsilon,
$$

and the conclusion follows in this case too.

Case 3: $C$ is a singleton; we can clearly assume $C=\{1\}$. We will argue as in Case 2. If we assume $x^{*}\left(x_{1}\right)>1-\delta$ we take $v_{1}=x_{1} \chi_{P_{1}}+\left(1-\left\|x_{1} \chi_{P_{1}}\right\|\right) e_{1}$. Then $v_{1}$ is a nonnegative element of the sphere of $\ell_{1}^{n}$ such that $\left\|v_{1}-x_{1}\right\|<$ $2 \delta<\varepsilon$. If $\left\|v_{1}+x_{2}-x_{3}\right\| \leq 1$ we get the conclusion. On the other hand, if $a=\left\|v_{1}+x_{2}-x_{3}\right\|>1$, we have $a<1+2 \delta$, and so $1-1 / a<2 \delta$. Then by taking $z_{1}=v_{1} / a+(1-1 / a) e_{1}, z_{2}=x_{2} / a$ and $z_{3}=x_{3} / a+(1-1 / a) e_{1}$, we find that $z_{i} \in B_{\ell_{1}^{n}}$ for every $i \leq 3, x^{*}\left(z_{1}\right)=1$,

$$
\left\|z_{1}+z_{2}-z_{3}\right\|=\frac{1}{a}\left\|v_{1}+x_{2}-x_{3}\right\|=1
$$

and also

$$
\left\|z_{1}-x_{1}\right\|<6 \delta<\varepsilon, \quad\left\|z_{2}-x_{2}\right\|<2 \delta<\varepsilon, \quad\left\|z_{3}-x_{3}\right\|<4 \delta<\varepsilon .
$$

We have proved that $\ell_{1}^{n}$ satisfies the AHSP- $\ell_{\infty}^{3}$ with $\delta(\varepsilon)=\varepsilon / 48$. 
Theorem 2.7. Let $X$ be a Banach space such that $X=\overline{\bigcup\left\{Y_{\alpha}: \alpha \in \Lambda\right\}}$, where $\left\{Y_{\alpha}: \alpha \in \Lambda\right\}$ is a nested family of subspaces of $X$ satisfying the AHSP- $\ell_{\infty}^{3}$ with the same function $\delta$. Then $X$ has the $A H S P-\ell_{\infty}^{3}$.

Proof. By assumption and the proof of Proposition 2.2, we can assume that there is a function $\eta$ satisfying (3) in Proposition 2.2 for each subspace $Y_{\alpha}$.

Assume that $\left\{x_{i}: i \leq 3\right\} \subset B_{X}$ satisfies $\left\|x_{1}+x_{2}-x_{3}\right\| \leq 1$ and also for some element in the convex hull of $\left\{x_{i}: i \leq 3\right\}$ we have

$$
\left\|\sum_{i=1}^{3} \alpha_{i} x_{i}\right\|>1-\eta\left(\frac{\varepsilon}{2}\right) .
$$

Choose $t>0$ such that

$$
t<\frac{1}{4} \min \left\{\frac{\varepsilon}{2},\left\|\sum_{i=1}^{3} \alpha_{i} x_{i}\right\|-1+\eta\left(\frac{\varepsilon}{2}\right)\right\} .
$$

Since $X$ is the closure of a nested family of subspaces, there exist $Y=Y_{\alpha} \subset X$ and $y_{i} \in B_{Y}$ for $i \leq 3$ satisfying

$$
\left\|y_{i}-x_{i}\right\|<t \quad \text { for all } i \leq 3 \text {. }
$$

Hence we have

$$
\left\|\sum_{i=1}^{3} \alpha_{i} y_{i}\right\|>\left\|\sum_{i=1}^{3} \alpha_{i} x_{i}\right\|-\max \left\{\left\|x_{i}-y_{i}\right\|: i \leq 3\right\}>1-\eta\left(\frac{\varepsilon}{2}\right) .
$$

If additionally $\left\|y_{1}+y_{2}-y_{3}\right\| \leq 1$, then we are done since $Y$ satisfies the $\mathrm{AHSP}-\ell_{\infty}^{3}$

Otherwise,

$$
1<a:=\left\|y_{1}+y_{2}-y_{3}\right\| \leq\left\|x_{1}+x_{2}-x_{3}\right\|+\sum_{i=1}^{3}\left\|x_{i}-y_{i}\right\|<1+3 t
$$

and so

Since for every $i \leq 3$ we have

$$
\left\|\frac{y_{1}}{a}+\frac{y_{2}}{a}-\frac{y_{3}}{a}\right\|=1 .
$$

$$
\left\|y_{i}-\frac{y_{i}}{a}\right\| \leq 1-\frac{1}{a}<3 t,
$$

it follows that

$$
\left\|x_{i}-\frac{y_{i}}{a}\right\| \leq\left\|x_{i}-y_{i}\right\|+\left\|y_{i}-\frac{y_{i}}{a}\right\|<t+3 t=4 t<\frac{\varepsilon}{2} .
$$


We also have

$$
\left\|\sum_{i=1}^{3} \alpha_{i} \frac{y_{i}}{a}\right\| \geq\left\|\sum_{i=1}^{3} \alpha_{i} x_{i}\right\|-\max \left\{\left\|x_{i}-\frac{y_{i}}{a}\right\|: i \leq 3\right\}>1-4 t>1-\eta\left(\frac{\varepsilon}{2}\right) .
$$

By Proposition 2.2 there are $A \subset\{1,2,3\}, z_{i} \in B_{Y}$ and $y^{*} \in S_{Y^{*}}$ such that

(i) $\sum_{i \in A} \alpha_{i}>1-\varepsilon / 2$,

(ii) $\left\|z_{i}-y_{i} / a\right\|<\varepsilon / 2$ for every $i \leq 3$,

(iii) $y^{*}\left(z_{i}\right)=1$ for all $i \in A$,

(iv) $\left\|z_{1}+z_{2}-z_{3}\right\| \leq 1$.

Hence for every $i \leq 3$ we also obtain

$$
\left\|z_{i}-x_{i}\right\| \leq\left\|z_{i}-\frac{y_{i}}{a}\right\|+\left\|\frac{y_{i}}{a}-x_{i}\right\|<\frac{\varepsilon}{2}+\frac{\varepsilon}{2}=\varepsilon .
$$

If we consider a Hahn-Banach extension $x^{*}$ of the functional $y^{*}$ to $X$, our conclusion follows. By Proposition 2.2, $X$ has the AHSP- $\ell_{\infty}^{3}$.

Let us remark that the subspace of $L_{1}(\mu)$ generated by a finite number of characteristic functions is linearly isometric to some space $\ell_{1}^{n}$. Since the space of simple functions is dense in $L_{1}(\mu)$, in view of Proposition 2.6, we conclude that $L_{1}(\mu)$ satisfies the assumption of the previous result. Hence we deduce the following assertion.

Proposition 2.8. The space $L_{1}(\mu)$ has the approximate hyperplane sum property for $\ell_{\infty}^{3}$ for any positive measure $\mu$.

If $Y$ has the AHSP- $\ell_{\infty}^{3}$, then it is immediate that $Y$ has the approximate hyperplane series property for convex combinations of two elements. By the proof of $\left[2\right.$, Theorem 4.1] the pair $\left(\ell_{1}^{2}, Y\right)$ has the BPBp. Hence so does $\left(\ell_{\infty}^{2}, Y\right)$. Now we prove the main result.

Theorem 2.9. $\left(\ell_{\infty}^{3}, Y\right)$ has the BPBp if and only if $Y$ has the $A H S P-\ell_{\infty}^{3}$.

Proof. Given $0<\varepsilon<1$, assume that $\left(\ell_{\infty}^{3}, Y\right)$ has the BPBp with a function $\eta(\varepsilon)$. We choose $0<\delta<\varepsilon / 6$.

Let $\left\{y_{k}: k \leq 3\right\}$ be a subset of $B_{Y}$ and consider nonnegative real numbers $\left\{\alpha_{k}: 1 \leq k \leq 3\right\}$ with $\sum_{k=1}^{3} \alpha_{k}=1$ satisfying $\left\|y_{1}+y_{2}-y_{3}\right\| \leq 1$ and

$$
\left\|\sum_{k=1}^{3} \alpha_{k} y_{k}\right\|>1-\min \{\delta, \eta(\delta)\}
$$


We write $u_{1}=(-1,1,1), u_{2}=(1,-1,1), u_{3}=(1,1,1)$ and $u_{4}=(-1,-1,1)=$ $u_{1}+u_{2}-u_{3}$ and

$$
x=\alpha_{1} u_{1}+\alpha_{2} u_{2}+\alpha_{3} u_{3}=\left(-\alpha_{1}+\alpha_{2}+\alpha_{3}, \alpha_{1}-\alpha_{2}+\alpha_{3}, 1\right) .
$$

Consider the linear operator $T: \ell_{\infty}^{3} \rightarrow Y$ that satisfies $T\left(u_{i}\right)=y_{i}$ for every $i \leq 3$. Since the unit ball of $\ell_{\infty}^{3}$ is the absolutely convex hull of $\left\{u_{i}: i \leq 4\right\}$, by assumption we know that $0<\|T\| \leq 1$, and also

$$
\left\|\frac{T x}{\|T\|}\right\| \geq\|T x\|>1-\min \{\delta, \eta(\delta)\} .
$$

Hence there exist $S: \ell_{\infty}^{3} \rightarrow Y, z \in S_{\ell_{\infty}^{3}}$ and $y^{*} \in S_{Y^{*}}$ such that

$$
y^{*} S z=\|S\|=1, \quad\left\|S-\frac{T}{\|T\|}\right\|<\delta \quad \text { and } \quad\|z-x\|<\delta
$$

and so

$$
\|z-x\|<3 \delta
$$

We now show that there exists a subset $A \subset\{1,2,3\}$ such that the functional $y^{*}$ and the elements $v_{i}=S\left(u_{i}\right)$ for $i \leq 3$ satisfy condition (3) in Proposition 2.2. First, notice that for any $i \leq 3$ we have

$$
\begin{aligned}
\left\|v_{i}-y_{i}\right\| & =\left\|S\left(u_{i}\right)-T\left(u_{i}\right)\right\| \leq\left\|S\left(u_{i}\right)-\frac{T\left(u_{i}\right)}{\|T\|}\right\|+\left\|\frac{T\left(u_{i}\right)}{\|T\|}-T\left(u_{i}\right)\right\| \\
& <\delta+1-\|T\|<2 \delta<\varepsilon,
\end{aligned}
$$

so assertion (ii) of (3) in Proposition 2.2 is satisfied.

We will use the functionals $\left\{u_{i}^{*}: 1 \leq i \leq 3\right\} \subset S_{\left(\ell_{\infty}^{3}\right) *}$ given by

$$
u_{1}^{*}=\frac{1}{2}\left(-e_{1}^{*}+e_{3}^{*}\right), \quad u_{2}^{*}=\frac{1}{2}\left(-e_{2}^{*}+e_{3}^{*}\right), \quad u_{3}^{*}=\frac{1}{2}\left(e_{1}^{*}+e_{2}^{*}\right) .
$$

Clearly, $u_{i}^{*}\left(u_{j}\right)=\delta_{i}^{j}$ for any $i, j \leq 3$.

We claim that we may assume $z$ is the convex combination $\sum_{i=1}^{3} \beta_{i} u_{i}$.

Indeed, since we know that $z=\left(z_{1}, z_{2}, z_{3}\right)$ satisfies $z_{3} \neq-1, z$ is a convex combination of $\left(z_{1}, z_{2}, 1\right)$ and $\left(z_{1}, z_{2},-1\right)$ and $\left\|\left(z_{1}, z_{2}, 1\right)-x\right\| \leq\|z-x\|$, so we may assume $z=\left(z_{1}, z_{2}, 1\right)$. Hence, we can write $z$ as a convex combination $\beta_{1} u_{1}+$ $\beta_{2} u_{2}+\beta_{3} u_{3}$ or $z=\beta_{1} u_{1}+\beta_{2} u_{2}+\beta_{4} u_{4}$, where $\beta_{4}>0$.

Assume that $\beta_{4}>0$. By using

$$
\alpha_{3}+\beta_{4}=u_{3}^{*}(x-z) \leq\|x-z\|<\delta,
$$


we obtain $\beta_{4}<\delta$. Hence $u=\frac{\beta_{1} u_{1}+\beta_{2} u_{2}}{\beta_{1}+\beta_{2}}$ clearly satisfies $y^{*}(S u)=1$ and also

$$
\begin{aligned}
\|u-x\| & \leq\|u-z\|+\|z-x\| \leq\left\|u-\left(\beta_{1} u_{1}+\beta_{2} u_{2}\right)\right\|+\left\|\beta_{4} u_{4}\right\|+\|z-x\| \\
& \leq\left(\frac{1}{\beta_{1}+\beta_{2}}-1\right)\left(\beta_{1}+\beta_{2}\right)+\beta_{4}+\delta=2 \beta_{4}+\delta<3 \delta .
\end{aligned}
$$

So we can use $u$ instead of $z$, and $u$ belongs to the convex hull of $\left\{u_{i}: i \leq 2\right\}$ and satisfies the estimate (2.9).

Hence we may assume that $z=\sum_{i=1}^{3} \beta_{i} u_{i}$. We take

$$
A=\left\{i \leq 3: \beta_{i} \neq 0\right\} \text {. }
$$

Since for every $i \leq 3$ we have

$$
\left|\beta_{i}-\alpha_{i}\right|=\left|u_{i}^{*}(z-x)\right| \leq\|z-x\|<3 \delta,
$$

we obtain $\alpha_{i}<3 \delta$ for every $i \in\{1,2,3\} \backslash A$ and so

$$
\sum_{i \in A} \alpha_{i}>1-6 \delta>1-\varepsilon
$$

hence condition (i) in (3) of Proposition 2.2 is satisfied. Conditions (iii) and (iv) there also hold for the functional $y^{*}$ and the vectors $v_{k}=S\left(u_{k}\right)(1 \leq k \leq 3)$.

Assume now that $Y$ has the AHSP- $\ell_{\infty}^{3}$. Assume that condition (3) in Proposition 2.2 is satisfied for a function $\eta(\varepsilon)$. Given $0<\varepsilon<1 / 2$, assume that $T \in$ $S_{L\left(\ell_{\infty}^{3}, Y\right)}$ and $x \in S_{\ell_{\infty}^{3}}$ satisfy

$$
\|T x\|>1-\eta(\varepsilon) .
$$

Since $x$ is in $S_{\ell_{\infty}^{3}}$, it is a convex combination of three extreme points of $B_{\ell_{\infty}^{3}}$ that belong to the same face of the unit ball. By composing with a convenient isometry, we may assume that these extreme points are $u_{1}=(-1,1,1), u_{2}=(1,-1,1)$ and $u_{3}=(1,1,1)$. Assume that $x$ is the convex combination

$$
x=\alpha_{1} u_{1}+\alpha_{2} u_{2}+\alpha_{3} u_{3} .
$$

Since $T \in S_{L\left(\ell_{\infty}^{3}, Y\right)}$, it is clear that $y_{1}=T u_{1}, y_{2}=T u_{2}$ and $y_{3}=T u_{3}$ satisfy the assumption of condition (3) in Proposition 2.2. Hence there are $A \subset\{1,2,3\}$, $\left\{z_{k}: k \leq 3\right\} \subset B_{Y}$ and $y^{*} \in S_{Y^{*}}$ satisfying conditions (i) to (iv) there.

Consider the linear operator $S: \ell_{\infty}^{3} \rightarrow Y$ satisfying $S u_{1}=z_{1}, S u_{2}=z_{2}$ and $S u_{3}=z_{3}$. We already know that $\left\{z_{k}: 1 \leq k \leq 3\right\}$ is contained in $B_{Y}$. Moreover in view of condition (iv) the element $S\left(u_{4}\right)=z_{1}+z_{2}-z_{3}$ belongs to $B_{Y}$ too. Thus, as above, $\|S\| \leq 1$. From condition (iii) we obtain

$$
1=y^{*}\left(S\left(\sum_{i \in A} \frac{\alpha_{i}}{\sum_{j \in A} \alpha_{j}} u_{i}\right) \leq\left\|S\left(\sum_{i \in A} \frac{\alpha_{i}}{\sum_{j \in A} \alpha_{j}} u_{i}\right)\right\| \leq\|S\| \leq 1,\right.
$$


so $S$ is in $S_{L\left(\ell_{\infty}^{3}, Y\right)}$ and attains its norm at $v=\sum_{i \in A} \frac{\alpha_{i}}{\sum_{j \in A} \alpha_{j}} u_{i}$. We also obtain

$$
\|S-T\|<3 \varepsilon \text { and } \quad\|v-x\| \leq 2 \varepsilon
$$

Hence the pair $\left(\ell_{\infty}^{3}, Y\right)$ has the Bishop-Phelps-Bollobás property for operators.

In view of the previous characterization and known results providing pairs of Banach spaces satisfying the BPBp, we already know the following examples of spaces with the AHSP- $\ell_{\infty}^{3}$ :

- Finite-dimensional spaces [2, Proposition 2.4].

- Spaces with the property $\beta$ of Lindenstrauss [2, Theorem 2.2].

Let us remark that not every Banach space has the AHSP- $\ell_{\infty}^{3}$.

Proposition 2.10. A strictly convex Banach space has the AHSP- $\ell_{\infty}^{3}$ if and only if it is uniformly convex.

Proof. Assume that a Banach space $X$ has the AHSP- $\ell_{\infty}^{3}$. Given $0<\varepsilon<1$, consider $x, y \in B_{X}$ such that $\|(x+y) / 2\|>1-\eta(\varepsilon / 2)$. We take $x_{1}=x, x_{2}=y$, $x_{3}=0, \alpha_{1}=\alpha_{2}=1 / 2$ and $\alpha_{3}=0$. By assumption there exist $A \subset\{1,2,3\}$, $\left\{z_{i}: i \leq 3\right\} \subset B_{X}$ and $x^{*} \in S_{X^{*}}$ such that

(i) $\sum_{i \in A} \alpha_{i}>1-\varepsilon / 2>1 / 2$,

(ii) $\left\|z_{i}-x_{i}\right\|<\varepsilon / 2$ for all $i \leq 3$,

(iii) $x^{*}\left(z_{i}\right)=1$ for all $i \in A$,

(iv) $\left\|z_{1}+z_{2}-z_{3}\right\| \leq 1$.

By (i) we have $A \supset\{1,2\}$. In view of (iii) we deduce that $z_{1}, z_{2} \in S_{X}$ and $\left\|z_{1}+z_{2}\right\|=2$. As every point of $S_{X}$ is an extreme point, we get $z_{1}=z_{2}$. Hence, by (ii) we get $\|x-y\|<\varepsilon$. It follows that $X$ is uniformly convex with modulus of convexity $\delta(\varepsilon) \geq \eta(\varepsilon / 2)$.

Very recently, in [10, Proposition 6], it has been shown that if $Y$ is a strictly convex space without the approximation property, then there exist an infinitedimensional subspace $X$ of $c_{0}$ and a compact operator $T$ from $X$ into $Y$ such that $T$ cannot be approximated by norm attaining operators. This gives a wealth of couples $(X, Y)$ failing the BPBp. Proposition 2.10 shows that it is possible to remove the hypothesis of infinite-dimensionality for $X$. More precisely, if $Y$ is a strictly convex space which is not uniformly convex, then $\left(\ell_{\infty}^{3}, Y\right)$ does not have the BPBp. 


\section{Acknowledgements}

The first author was supported MTM2012-31755, Junta de Andalucía P09-FQM4911 and FQM-185. The second author was supported by MTM2011-23843 and Junta de Andalucía grants FQM-199 and FQM-3737. The third and fifth authors were supported by MICINN MTM2011-22417 and by Prometeo II/2013/013. The fourth author was partially supported by Basic Science Research Program through the National of Korea (NRF) funded by the Ministry of Education, Science and Technology (2014R1A1A2056084).

\section{References}

[1] M. D. Acosta, The Bishop-Phelps-Bollobás property for operators on $\mathcal{C}(K)$, arXiv:1405.6428.

[2] M. D. Acosta, R. M. Aron, D. García and M. Maestre, The Bishop-Phelps-Bollobás theorem for operators, J. Funct. Anal. 254 (2008), 2780-2799. Zbl 1152.46006 MR 2414220

[3] R. M. Aron, B. Cascales and O. Kozhushkina, The Bishop-Phelps-Bollobás theorem and Asplund operators, Proc. Amer. Math. Soc. 139 (2011), 3553-3560. Zbl 1235.46013 MR 2813386

[4] B. Cascales, A. J. Guirao and V. Kadets, A Bishop-Phelps-Bollobás type theorem for uniform algebras, Adv. Math. 240 (2013), 370-382. Zbl 1298.46010 MR 3046314

[5] E. Bishop and R. R. Phelps, A proof that every Banach space is subreflexive, Bull. Amer. Math. Soc. 67 (1961), 97-98. Zbl 0098.07905 MR 0123174

[6] B. Bollobás, An extension to the theorem of Bishop and Phelps, Bull. London Math. Soc. 2 (1970), 181-182. Zbl 0217.45104 MR 0267380

[7] F. F. Bonsall and J. Duncan, Numerical ranges II, London Math. Soc. Lecture Note Ser. 10, Cambridge Univ. Press, Cambridge, 1973. Z Zbl 0262.47001 MR 0442682

[8] S. K. Kim, The Bishop-Phelps-Bollobás theorem for operators from $c_{0}$ to uniformly convex spaces, Israel J. Math. 197 (2013), 425-435. Zbl 1296.46008 MR 3096622

[9] S. K. Kim and H. J. Lee, The Bishop-Phelps-Bollobás property for operators from $\mathcal{C}(K)$ to uniformly convex spaces, J. Math. Anal. Appl. 421 (2015), 51-58. Zbl 06334520 MR 3250465

[10] M. Martín, Norm-attaining compact operators, J. Funct. Anal. 267 (2014), 1585-1592. Zbl 06320731 MR 3229801 